\title{
Atuação do enfermeiro na promoção e manejo do aleitamento materno: vivências de um acadêmico de enfermagem
}

Nurse's activities in the promotion and management of breastfeeding: the living of an academic of nursing

Papel de las enfermeras en la promoción y gestión de la lactancia materna: experiencias de una estudiante de enfermería

Gisele Batista de Oliveira

ORCID: https://orcid.org/0000-0002-9578-5650

Centro Universitário do Norte, Brasil

E-mail: giselebatistaoliver@hotmail.com

Orácio Carvalho Ribeiro Júnior

ORCID: https://orcid.org/0000-0003-3212-9936

Universidade do Estado do Pará, Brasil

E-mail: oracio.junior@uepa.br

Ruan Victor dos Santos Silva

ORCID: https://orcid.org/0000-0001-9754-3877

Centro Universitário do Norte, Brasil

E-mail: ruanenfermeiro02@gmail.com

Kaio Vinícius Paiva Albarado

ORCID: https://orcid.org/0000-0002-0687-7124

Universidade Federal do Oeste do Pará, Brasil

E-mail: kaioalbarado@gmail.com

Semírames Cartonilho de Souza Ramos

ORCID: https://orcid.org/0000-0001-8370-5994

Universidade Federal da Paraíba, Brasil

E-mail: semiramesramosmoga@gmail.com

Tayane Moura Martins

ORCID: https://orcid.org/0000-0003-3236-8574

Universidade Federal do Pará, Brasil

E-mail: tayane_m.martins1@hotmail.com

Amanda Caroline Duarte Ferreira

ORCID: https://orcid.org/0000-0002-1071-597X Faculdade de Ciências Humanas e Sociais do Xingu e Amazônia, Brasil

E-mail: amandacdf1125@gmail.com

Higor Barbosa da Silva

ORCID: https://orcid.org/0000-0003-2110-8988

Distrito Sanitário Especial Indígena de Altamira, Brasil

E-mail: enf.higor@hotmail.com

Patrícia Resende Barbosa

ORCID: https://orcid.org/0000-0002-1821-0992

Distrito Sanitário Especial Indígena de Altamira, Brasil

E-mail: patricabarbosa@hotmail.com

Rosane Silva dos Santos

ORCID: https://orcid.org/0000-0002-2872-3505

Universidade do Estado do Pará, Brasil

E-mail: rosane.ssantos@ hotmail.com

Janete de Oliveira Briana

ORCID: https://orcid.org/0000-0001-6302-5035 Universidade do Estado do Pará, Brasil

E-mail: janetebriana@gmail.com

Suzana Maria da Silva Ferreira

ORCID: https://orcid.org/0000-0001-9774-4820

Universidade Federal de Roraima, Brasil

E-mail: suzana-ferreira@live.com

Aline Verçosa de Figueiredo

ORCID: https://orcid.org/0000-0001-8752-6432 Universidade do Estado do Pará, Brasil

E-mail: v.alinne@yahoo.com.br 


\author{
William Cardoso da Cunha \\ ORCID: https://orcid.org/0000-0001-7660-0879 \\ Hospital Geral de Altamira São Rafael, Brasil \\ E-mail: enferobwilliamcunha@gmail.com \\ Elielza do Socorro Silva do Amaral \\ ORCID: https://orcid.org/0000-0002-4145-2172 \\ Secretaria Municipal de Saúde de Altamira, Brasil \\ E-mail: babyvilua@yahoo.com
}

\begin{abstract}
Resumo
Objetivo: descrever as experiências de um acadêmico de enfermagem nas ações de promoção do aleitamento materno durante o estágio supervisionado em uma maternidade pública de Manaus. Descrição de experiência: Trata-se de um estudo descritivo, tipo relato de experiência, elaborado no contexto da disciplina Saúde da Mulher e Estágio Supervisionado. Resultado: O conhecimento do enfermeiro e da equipe de enfermagem sobre as vantagens da amamentação são primordiais, esse conhecimento pode determinar toda uma conduta profissional e interferir na busca de estratégias que visam à promoção e manejo adequado ao aleitamento materno. Considerações finais: Pode-se considerar que a atividade foi super exitosa, pois através da experiência vivida juntamente com as gestantes e puérperas foi possível uma maior percepção da extrema importância que tem a atuação do enfermeiro no aleitamento materno.
\end{abstract}

Palavras-chave: Enfermeiro; Aleitamento materno; Promoção da saúde; Manejo.

\begin{abstract}
Objective: to describe the experiences of a nursing student in the actions to promote breastfeeding during the supervised internship at a public maternity hospital in Manaus. Description of experience: This is a descriptive study, an experience report, elaborated in the context of the discipline Women's Health and Supervised Internship. Results: The knowledge of the nurse and the nursing team about the advantages of breastfeeding are paramount, this knowledge can determine all professional conduct and interfere in the search for strategies that aim at the promotion and proper management of breastfeeding. Final considerations: It can be considered that the activity was super successful, because through the experience lived together with the pregnant women and puerperas it was possible to gain a greater perception of the extreme importance of the nurse's role in breastfeeding.
\end{abstract}

Keywords: Nursing; Breastfeeding; Promotion in the Health; Management.

\title{
Resumen
}

Objetivo: describir las experiencias de una estudiante de enfermería en acciones de promoción de la lactancia materna durante una pasantía supervisada en una maternidad pública de Manaus. Descripción de la experiencia: Se trata de un estudio descriptivo, tipo relato de experiencia, desarrollado en el contexto de la disciplina de Salud de la Mujer y Pasantía Supervisada. Resultado: El conocimiento de las enfermeras y del personal de enfermería sobre las ventajas de la lactancia materna es fundamental, este conocimiento puede determinar toda una conducta profesional e interferir en la búsqueda de estrategias encaminadas a promover y gestionar una adecuada lactancia materna. Consideraciones finales: Se puede considerar que la actividad fue súper exitosa, pues a través de la experiencia vivida en conjunto con mujeres embarazadas y posparto, se logró una mayor percepción de la extrema importancia del rol de las enfermeras en la lactancia materna.

Palabras clave: Enfermera; Amamantamiento; Promoción de la salud; Gestión.

\section{Introdução}

O aleitamento materno é uma prática que traz inúmeras vantagens para a mãe e o recém-nascido, apresentando alto impacto na redução da morbimortalidade infantil. O leite materno é capaz de suprir todas as necessidades nutricionais da criança durante os seis primeiros meses de vida, além de atuar como um importante mecanismo de proteção contra diversos tipos de infecções e surgimento de doenças alérgicas. Em fases posteriores da vida a prática da amamentação também deixa suas marcas benéficas, atuando como um fator de proteção ao risco de desenvolvimento de doenças cardiovasculares e obesidade (Aguiar \& Silva, 2016). O leite materno é a principal fonte de alimento das crianças; nele estão contidos nutrientes que são imprescindíveis para a proteção da saúde dos infantes contra infecções, diarreias, doenças respiratórias, alergias, entre outras. O crescimento e o desenvolvimento dos lactentes dependem significativamente das propriedades nutricionais e imunológicas que somente o leite materno oferece (Lima, Nascimento \& Martins, 2018). 
É consenso que o leite materno é o melhor alimento para a promoção e proteção da saúde da criança e apresenta reconhecidos benefícios nutricionais, imunológicos, cognitivos, econômicos, sociais e emocionais. Esses benefícios são aproveitados em sua magnitude quando o aleitamento materno (AM) é praticado exclusivamente até os seis meses de vida do bebê, permanecendo complementado por outros tipos de alimentos até os dois anos de idade ou mais, como recomenda a Organização Mundial de Saúde (OMS) (Cavalcanti et al. 2015).

Além disso, quando bem manejado, o aleitamento materno traz benefícios para a mãe e a criança que vão muito além daqueles observados durante o período neonatal. Para o lactente diminui a possibilidade de adoecimento, reduzindo as taxas de mortalidade infantil e as internações hospitalares, reduz ainda os riscos de doenças crônicas, a exemplo da obesidade. Além disso, a mãe tem como benefício, a involução uterina mais breve, redução na hemorragia uterina durante o pós-parto, perda de peso, diminuição dos riscos de câncer de mama e do colo do útero, sendo a alternativa mais econômica de alimentação do bebê, com forte impacto sobre a economia de uma sociedade que fortalece esta prática (Barbieri et al. 2016).

Segundo avaliação recente, a prevalência do AME (Aleitamento materno exclusivo) em crianças menores de 6 meses de vida, que constitui uma recomendação normativa, foi de apenas 37\% entre os anos de 2006 e 2010. Em outro estudo de base populacional houve prevalência de apenas 36,6 \% do aleitamento materno exclusivo até os 6 meses de idade e 45,4\% até o primeiro ano de vida (Boccolini et al. 2017). Por outro lado, estimativas sequenciais das pesquisas nacionais sobre demografia e saúde confirmaram uma tendência bem consolidada de aumento dessa prática no Brasil (Unicef, 2012).

Uma pesquisa do Instituto de Saúde da Secretaria Estadual de São Paulo (SES/SP) em 2010 observou que a prevalência do aleitamento materno exclusivo em menores de seis meses era de $41 \%$ no total das capitais brasileiras. Verificase que a região Norte foi a que apresentou maior prevalência desta prática (45,9\%), seguida das regiões Centro-Oeste (45,0\%), Sul $(43,9 \%)$ e Sudeste $(39,4 \%)$, e a região Nordeste foi a que apresentou indicadores mais baixos $(37,0 \%)$. Esses indicadores demonstram que a população brasileira estudada não conseguiu atingir a meta de 50\% de AME preconizada pela Organização Mundial de Saúde (OMS) (Brasil, 2010).

Apesar de todas as evidências científicas provando a superioridade da amamentação sobre outras formas de alimentar a criança pequena, e apesar dos esforços de diversos organismos nacionais e internacionais, as prevalências de aleitamento materno no Brasil, em especial as de amamentação exclusiva, estão bastante aquém das recomendadas, e o profissional de saúde tem papel fundamental na reversão desse quadro. Mas para isso, ele precisa estar preparado, pois, por mais competente que ele seja nos aspectos técnicos relacionados à lactação, o seu trabalho de promoção e apoio ao aleitamento materno não será bem-sucedido se ele não tiver um olhar atento, abrangente, sempre levando em consideração os aspectos emocionais, a cultura familiar, a rede social de apoio à mulher, entre outros. Esse olhar necessariamente deve reconhecer a mulher como protagonista do seu processo de amamentar, valorizando-a, escutando-a e emponderando-a (Brasil, 2015).

Em relação à atuação do enfermeiro no contexto da Política Nacional de Aleitamento Materno, ele deve estar preparado para prevenir, reconhecer e resolver as dificuldades na interação nutriz e filho, especialmente no que se refere à amamentação, como os obstáculos identificados para que a sua prática seja bem-sucedida. Portanto, é preciso ter um olhar atento para que essas necessidades da nutriz, durante o aleitamento no período de internação hospitalar, sejam precocemente identificadas e resolvidas, evitando o desmame precoce ou o início da alimentação complementar quando ainda se faz importante o aleitamento exclusivo. Assim, o manejo clínico da amamentação torna-se necessário para aprofundar a prática da amamentação e, do mesmo modo, intervir diretamente junto à nutriz para que ela seja capaz de prover uma alimentação saudável ao recém-nascido (Brasil, 2015).

Assim, este trabalho tem por objetivo descrever as experiências de um acadêmico de enfermagem nas ações de promoção do aleitamento materno durante o estágio supervisionado em uma maternidade pública de Manaus. 


\section{Metodologia}

Trata-se de um estudo qualitativo descritivo, observacional, tipo relato de experiência, elaborado no contexto da disciplina Saúde da Mulher e Estágio Supervisionado, ministrada no $7^{\circ}$ e $9^{\circ}$ período do curso de Graduação em Enfermagem do Centro Universitário do Norte (UNINORTE), que tem como objetivo principal descrever a atuação do enfermeiro na promoção e manejo da amamentação, assim como, abordar a dificuldade que muitas mães sentem para amamentar nesse primeiro momento logo após o parto e no decorrer do puerpério. A característica de estudos qualitativos tem por finalidade permitir a buscas por respostas de maneira mais subjetiva, analisando os fatos mais aprofundados de acordo com cada contexto (Teixeira, 2009).

Este estudo consiste em um relato de experiência vivenciado pelo docente e seus colegas de grupo em duas unidades de saúde distintas e em períodos distintos também na cidade de Manaus-Amazonas.

\section{Resultados}

A primeira experiência ocorreu no $7^{\circ}$ período do Curso de enfermagem do Centro Universitário do Norte (UNINORTE), na disciplina de Saúde da Mulher na Unidade de Saúde e Maternidade Instituto da Mulher Dona Lindu. Durante este estágio foi possível perceber na prática a grande dificuldade da maioria das puérperas para amamentar. E foi solicitado pela professora da disciplina que os acadêmicos elaborassem uma atividade de educação em saúde, atividade essa que foi inicialmente de aconselhamento de orientações. Estágio que foi elaborado no período de 04 de setembro de 2017 até 15 de setembro de 2017.

Elaborou-se então uma palestra educativa em cada enfermaria, onde passamos em todas as enfermarias com intuito de expor práticas educativas, com o tema amamentação. Fomos ao encontro das puérperas, e lá abordamos os benefícios da amamentação para a mães e seus acompanhantes, mostramos os modos correto como se deve amamentar, as melhores posições do RN e das mães para o melhor saída do leite materno, como segurar a mama nesse momento, falou-se também sobre massagens que podem ser realizadas nas mamas para facilitar a saída do leite, orientando as mães, as que não podem amamentar, em como deve ser realizado o procedimento, as mães que tem um processo de produção maior de leite materno nas mamas, podem se dirigir ao banco de leite humano para doação, para as mães que não podem amamentar por algumas dessas razões; mães ser portadora do vírus HIV, está em tratamento de tuberculose, mãe em tratamento de quimioterapia ou radioterapia, mães que fazem uso de medicamentos que afetam a criança, mães que fazem uso de entorpecentes.

A segunda experiência ocorreu no $9^{\circ}$ período do Curso de enfermagem do Centro Universitário do Norte (UNINORTE), na disciplina estágio supervisionado na Unidade Básica de Saúde Mansour Bubol. Na UBS realizamos uma atividade também de educação em saúde, onde o foco era as usuárias com acompanhamento de pré-natal, onde foi elaborado uma palestra sobre mitos e verdades da amamentação. Realizamos a atividade durante as consultas do pré-natal, percorremos toda a unidade básica de saúde, palestramos nos corredores, nos auditórios e abrimos a oportunidade para que fossem tiradas todas as dúvidas. As principais dúvidas apresentadas pelas puérperas sobre o manejo do aleitamento materno foram: existência de leite fraco, uso de pente nas mamas e seu benefício na saída do leite, existência de produtos naturais para a produção de leite, a influência da cerveja preta sobre a produção do leite, amamentação diante da presença de mamilos fissurados e manejo de mamas endurecidas. No período das 13:00h às 17:00h na data de 14 de agosto de 2018 até 05 de setembro de 2018.

Diante das dúvidas levantadas pelas usuárias, procedeu-se então às orientações para dirimir cada ponto levantado. Neste processo pode-se perceber momentos muito leigos por parte dos usuários, seguindo-se então as seguintes orientações: a organização de mundial de saúde, o ministério da Saúde, a sociedade brasileira de pediatria recomenda a importância do leite materno em demanda espontânea e exclusivo até o 6 mês, estendida até dois anos ou mais, a primeira mamada e a mais importante, o líquido chamado colostro, um líquido amarelo que e rico em vários nutrientes, é a primeira vacina do RN 
propriamente dita, e muito importante para o recém-nascido $(\mathrm{RN})$, e não existe leite fraco, cada mãe produz o leite que sua criança precisa, apenas 5 minutos de amamentação a criança já tem $70 \%$ da alimentação que ele precisa, o pente nas mamas e uma lenda antiga, muito usada pelas mães de antigamente, mães que tiveram que lhe dar com mamas ingurgitadas, hoje com novas pesquisas, é usadas as palmas das mãos ou poupas digitais das mãos em movimentos circulares, facilita no manejo na saída do leite, e hipótese nenhuma deve ser feito uso de bebidas alcoólicas de qualquer qualidade, para aquelas mães que desenvolve lesões em mamilos, tem que oferece a outra mama não lesionada, é muito importante não deixar de amamentar mesmo que a lesão esteja nas duas mamas, para evitar tipos de lesões e preciso que o mamilo da mãe esteja no palato da criança, e sempre a pega correta seja aplicada, com a boca da criança alcançando toda a auréola das mamas.

Ao final, percebeu-se que as informações foram muito importantes para prática educativa das usuárias com o intuito de prepará-las para a vivência do aleitamento materno de forma emponderada. Na sequência foi distribuído um folder educativo com orientações chaves sobre a prática e o manejo do aleitamento materno.

\section{Discussão}

O conhecimento do enfermeiro e da equipe de enfermagem sobre as vantagens da amamentação são primordiais, esse conhecimento pode determinar toda uma conduta profissional e interferir na busca de estratégias que visam à promoção e manejo adequado ao aleitamento materno.

A assistência do enfermeiro exerce grande importância no sucesso do aleitamento materno, sistematizando o atendimento realizado por meio de anamnese e exame físico, diagnóstico de enfermagem, implementação e evolução dos resultados. Os cuidados prestados devem estar de acordo com o problema encontrado, realizando intervenções necessárias para que as interferências no momento da amamentação possam ser minimizadas ou solucionadas. O enfermeiro deve estar presente, observando como está sendo a pega do recém-nascido, e instruindo a mãe quando surgir alguma dúvida quanto ao aleitamento materno e aos cuidados com o recém-nascido (Carvalho, Carvalho \& Magalhães, 2011).

Carvalho, Carvalho e Magalhães (2011) ainda afirmam que é necessária a comunicação clara e objetiva durante a orientação, o incentivo e o apoio ao aleitamento materno, demonstrando diversas posições, promovendo relaxamento e posicionamento confortáveis para o binômio, explicando a fonte dos reflexos da criança e mostrando como isso pode ser usado para ajudar na sucção do recém-nascido.

Entre os profissionais da Estratégia de Saúde da Família (ESF) o enfermeiro é o sujeito que deve estar preparado para lidar e direcionar uma demanda diversificada, principalmente quando se tratar de questões de ordem da mulher nutriz, pois deve ser capaz de identificar e oportunizar momentos educativos, facilitando a amamentação (Amorim \& Andrade, 2009).

No manejo clínico da amamentação, é necessário que o enfermeiro tenha conhecimento técnico e científico sobre anatomia e fisiologia da lactação, da sucção, dos fatores emocionais e psicológicos que possam interferir, além de técnicas de comunicação, para que saiba orientar sobre posicionamento e pega adequada, extração manual do leite materno e formas alternativas de oferta do leite materno, que não sejam por meio de mamadeiras (Azevedo et al. 2015).

Os profissionais de saúde têm papel fundamental, fornecendo informações desde o acompanhamento pré-natal mediante o apoio emocional e orientações do ponto de vista prático, possibilitando que as mulheres desenvolvam a autoconfiança em sua capacidade de amamentar, aprendam como superar dificuldades e experimentem êxitos com a amamentação no pós-parto imediato (Vieira et al. 2016).

As ações de promoção do aleitamento materno que poderão ser realizadas por enfermeiras de unidades de estratégia de saúde da família são importantes formas de incentivar e, consequentemente, elevar os índices de aleitamento materno. A consulta de pré-natal, os encontros com a comunidade, os grupos de gestantes e as consultas e visitas puerperais são formas de promover o aleitamento materno dentro da comunidade (Oliveira \& Gavasso, 2012). 
Para melhoria dos seus índices de aleitamento materno, faz-se necessário e adequado o aprendizado das mães com participação ativa dos profissionais de saúde, propiciando orientações e suporte oportunos para as gestantes e lactantes. Avaliar o conhecimento desses profissionais representa uma estratégia de reconhecimento do cenário de apoio à prática da amamentação (Caldeira et al. 2007).

As evidências científicas parecem apontar para a importância dos mitos e crenças como possíveis causas que justificam a complementação precoce, de acordo com as mães. Segundo essa lógica, os mitos e crenças sobre o aleitamento materno "leite fraco", "pouco leite", "o bebê não quis pegar o peito", "o leite materno não mata a sede do bebê" e "os seios caem com a lactação" evidenciam a insegurança da mulher ante questões do cotidiano materno durante a amamentação, tais como a produção de leite materno de qualidade e em quantidade suficiente para o bebê; o choro da criança que, em geral, é associado a fome; a época correta para a introdução de água e/ou outros líquidos para o bebê, dentre outros aspectos. Assim, essas representações muitas vezes justificam a introdução precoce de outros alimentos, a oferta de chupeta e mamadeira, ou mesmo a interrupção do aleitamento materno (Marques, Cotta \& Priore, 2011).

Conforme Vitolo et al. (2014) quando o aleitamento materno é fortemente inserido na cultura de um povo ou de uma região, esta prática é transmitida de geração a geração e a prevalência do aleitamento materno é maior, fazendo que com os seus benefícios também tenham a mesma perpetuação nos diferentes contextos e vertentes.

Na busca pela melhora dos índices de amamentação, muitas estratégias têm sido utilizadas nos serviços de saúde. Destacam-se nesse cenário as tecnologias como instrumento capaz de despertar o interesse dos usuários sobre a temática. As tecnologias podem ser definidas como um conjunto de saberes e fazeres pertinentes a produtos e materiais que determinam terapêuticas e processos de trabalho e estabelecem instrumentos para realizar ações na produção da saúde (Silva et al. 2016).

Dentre as tecnologias educacionais mais comumente encontradas estão as palestras, folhetos, cartões postais, livretos, filmagens durante o Aleitamento Materno, software educativo, vídeos sobre Aleitamento Materno, pôsteres, álbum seriado e aconselhamento. A utilização dessas tecnologias pelo profissional de saúde pode contribuir para a prática de orientação à mulher no puerpério, porém, o profissional de saúde deverá ter consciência de que ensinar não é transferir conhecimento e sim criar possibilidades para a sua produção e sua construção (Oliveira \& Pagliuca, 2013).

Com base em estudos, pode se referir como exemplos de ferramentas tecnológicas utilizadas na promoção ao aleitamento materno: Álbum seriado, tecnologia digital, com educação a distância, tecnologia da videoconferência e a tecnologia do CD ROOM com informações relevantes para as mães.

Os manuais educativos são utilizados como estratégia de educação em saúde, devem ser fundamentados em termos científicos, e conterem propostas de atividades para recuperar e desenvolver aptidões físicas, mentais e sociais, uniformizando as orientações para facilitar o trabalho da equipe multidisciplinar com vistas ao cuidado em saúde (Leite et al. 2018).

O álbum seriado é uma ferramenta utilizada nas unidades de saúde para a promoção em saúde e consiste em ilustrações e textos. Quando utilizado na unidade de saúde hospitalar logo após o parto, favorece a autoeficácia materna, sobretudo entre as puérperas de 20 a 29 anos, casadas ou que vivem em união consensual e que se encontram empregadas. (Dodt et al. 2013).

A Educação a Distância é uma ferramenta estratégica importante na qualificação dos profissionais para atuarem no mundo da informação, sendo uma tarefa complexa. O Programa Nacional de Telessaúde foi instituído no Brasil em 2007 com o "objetivo de desenvolver ações de apoio à assistência à saúde e, sobretudo, de educação permanente de Saúde da Família", proporcionando aos profissionais, informações sobre amamentação nos diversos aspectos (Brasil, 2015).

A videoconferência é uma tecnologia inovadora que aumenta a acessibilidade ao serviço de saúde. Ela pode ser utilizada em tempo real, conectando consultores em lactação às mães que amamentam em seu próprio domicílio. Algumas 
limitações com a transmissão da tecnologia, como o áudio e vídeo podem comprometer o aleitamento materno e levar ao desmame precoce (Silva et al. 2016).

\section{Considerações Finais}

Pode-se considerar que a atividade foi super exitosa, pois através da experiência vivida juntamente com as gestantes e puérperas foi possível uma maior percepção da extrema importância que tem a atuação do enfermeiro para possibilitar de fato a promoção e um melhor manejo do aleitamento materno. Além de que, através de um bom relacionamento do enfermeiro com as pacientes e familiares, o aumento de confiança entre as partes, fez surgir um maior empoderamento das mulheres nessa fase tão importante da vida da mãe, bebê e família num todo.

Vale ressaltar que para a mulher executar as orientações do enfermeiro, ela precisa confiar nele, acreditar em suas palavras e para isso é necessário que haja um vínculo entre eles, que o enfermeiro seja simpático, saiba fazer uma boa abordagem e além de tudo, que ele transmita domínio sobre o assunto e segurança ao repassar as informações.

Como acadêmico de enfermagem foi muito importante o contato direto com a rotina nas unidades de saúde, ver na prática como acontece na realidade, ver as principais dificuldades que as mulheres enfrentam com relação à amamentação e auxiliar referente aos muitos mitos e verdades referentes ao assunto. Adquiri um conhecimento excelente, foi uma experiência enriquecedora para minha vida acadêmica e quero levar comigo para o decorrer de minha vida profissional.

Apesar de toda a facilidade de informação ainda existe muita dificuldade em grande parte das mulheres com relação à amamentação, e cabe ao profissional enfermeiro auxiliar nesse momento, e para isso é necessário um preparo adequado e por conta disso é extremamente importante se abordar o assunto da promoção e manejo do aleitamento materno.

Nesse contexto, estudos de cunho quantitativo que possam mensurar o grau de entendimento das mães sobre o aleitamento materno, se tronam pertinentes. Essa sugestão parte da premissa de que a enfermagem pode atuar com base em evidências, permitindo melhor atuação desses profissionais com relação ao aleitamento materno, além de difundir o conhecimento nessa área.

\section{Referências}

Aguiar, H., \& Silva, A. I. (2011). Aleitamento materno: a importância de intervir. Revista Acta Médica Portuguesa. 24(4):889-96. https://www.actamedicaportuguesa.com/revista/index.php/amp/article/view/1581/1164.

Amorim, M. M., Andrade, E. R. (2009). Atuação do enfermeiro no PSF sobre aleitamento materno. Revista Perspectivas online. 3 (9):93-110. http://www.perspectivasonline.com.br/edicao.php?\&numero=9>.

Azevedo, A. R. R., Alves, V. H., Souza, R. M. P., Rodrigues, D. P., Branco, M. B. L. R., \& Cruz, A. F. N. (2015). O manejo clínico da amamentação: saberes dos enfermeiros. Escola Anna Nery Revista de Enfermagem. 19(3): 439-445. 10.5935/1414-8145.20150058

Barbieri, M. C., Bercini, L. O., Brondani, K. J. M., Ferrari, R. A. P., Tacla, M. T. G. M., \& Sant'anna, F. L.(2015). Aleitamento materno: orientações recebidas no pré-natal, parto e puerpério. Semina: Ciências Biológicas e da Saúde. 36(1):17-24.doi: 10.5433/1679-0367.2014v35n2p17.

Boccolini, C. S., Boccolini, P. M. M., Monteiro, F. R., Venâncio, S. I., \& Giugliani, E. R. J. (2017). Tendência de indicadores do aleitamento materno no Brasil em três décadas. Revista de Saúde Pública.51:108. https://doi.org/10.11606/S1518-8787.2017051000029

Brasil, Ministério da Saúde (BR). Pesquisa de prevalência de aleitamento materno em municípios brasileiros. Ministério da saúde; 2010. http://www.redeblh.fiocruz.br/media/pamuni.pdf.

Brasil. Ministério da Saúde. Secretaria de Atenção à Saúde. Departamento de Atenção Básica. Saúde da criança: aleitamento materno e alimentação complementar / Ministério da Saúde, Secretaria de Atenção à Saúde, Departamento de Atenção Básica. (2a ed.), 2015. (Cadernos de Atenção Básica ; n. 23). http://bvsms.saude.gov.br/bvs/publicacoes/saude_crianca_nutricao_aleitamento_alimentacao.pdf.

Caldeira, A. P., Aguiar, G. N., Magalhães, W. A. C., \& Fagundes, G. C. (2007). Conhecimentos e práticas de promoção do aleitamento materno em Equipes de Saúde da Família em Montes Claros, Minas Gerais, Brasil. Cadernos de Saúde Pública. 23(8):1965-1970. https://doi.org/10.1590/S0102311 X2007000800023. 
Research, Society and Development, v. 10, n. 10, e227101018615, 2021

(CC BY 4.0) | ISSN 2525-3409 | DOI: http://dx.doi.org/10.33448/rsd-v10i10.18615

Carvalho, J. K. M.; Carvalho, C. G.; \& Magalhães, S. R. (2011). Importância da assistência de enfermagem no aleitamento materno. e-Scientia. 4 (2): 11-20. https://revistas.unibh.br/dcbas/article/view/186.

Cavalcanti, S. H., Caminha, M. de F. C., Figueiroa, J. N., Serva, V. M. S. B. D., Cruz, R. S de. B. L. C., Lira, P. I. C., \& Filho, M. B. (2015). Fatores associados à prática do aleitamento materno exclusivo por pelo menos seis meses no estado de Pernambuco. Revista Brasileira de Epidemiologia. 18(1): 20819. DOI: $10.1590 / 1980-5497201500010016$

Dodt, R. C. M., Ferreira, A. M. V., Nascimento, L. A., Macêdo, A. C., Joventino, E. S., \& Ximenes, L. B. (2013). Influência de estratégia de educação em saúde mediada por álbum seriado sobre a autoeficácia materna para amamentar. Texto \& Contexto Enfermagem. 22(3):610-8. http://www.scielo.br/scielo.php?script=sci_arttext\&pid=S0104-07072013000300006.

Leite, S. S., Afio, A. C. E., Carvalho, L. V., Silva, J. M., Almeida, P. C., \& Pagliuca, L. M. F. (2018). Construction and validation of na Educational Content Validation Instrument in Health. Revista Brasileira de Enfermagem. 71(4):1635-41. http://dx.doi.org/10.1590/0034-7167-2017-0648

Lima, A. P. C., Nascimento, D. da S., \& Martins, M. M. F.(2018). A prática do aleitamento materno e os fatores que levam ao desmame precoce: uma revisão integrativa. Journal of Health and Biological Sciences. 6 (2): 189-196. 10.12662/2317-3076jhbs.

Marques, E. S., Cotta, R. M. M., \& Priore, S. E. (2011). Mitos e crenças sobre o aleitamento materno. Ciência \& Saúde Coletiva. 16(5):2461-2468. https://doi.org/10.1590/S1413-81232011000500015.

Oliveira, A. P de; \& Gavasso, W. C. (2012). A atuação do enfermeiro na promoção do aleitamento materno em unidades de estratégia de saúde da família do município de Joaçaba, SC. Unoesc \& Ciência - ACBS. 3 (1):7-16.https://editora.unoesc.edu.br/index.php/acbs/article/view/1296/pdf.

Oliveira, P. M., \& Pagliuca, L. M. F.(2013). Avaliação de tecnologia educativa na modalidade literatura de cordel sobre amamentação. Revista da Escola de Enfermagem da USP. 47(1):205- 12. http://www.scielo.br/pdf/reeusp/v47n1/a26v47n1.pdf.

Silva, A. M. C. e., Freitas, L. M. C., Maia, J. A. de F., Granja, M. M. F., Dodt, R. C. M., \& Chaves, E. M. C. (2016). Tecnologias em aleitamento materno: revisão integrativa. Revista Brasileira em Promoção da Saúde. 29(3): 439-446. http://periodicos.unifor.br/RBPS/article/view/4812/pdf.

Teixeira, E. (2009). As Três Metodologias: acadêmica, da ciência e da pesquisa. Vozes.

Vieira, T. O., Martins, C. C., Santana, G. S., Vieira, G. O., \& Silva, L. R. (2016). Intenção materna de amamentar: revisão sistemática. Revista Ciência e Saúde Coletiva. 21 (12): 3845-3858. 10.1590/1413-812320152112.17962015.

Vítolo, M. R., Louzada, M. L., Rauber, F., Grechi, P., \& Gama, C. M. (2014). Impacto da atualização de profissionais de saúde sobre as práticas de amamentação e alimentação complementar. Cadernos de Saúde Pública. 30(8):1695-1707. http://dx.doi.org/10.1590/0102-311X00186913.

Unicef. Fundo das Nações Unidas para a Infância. Situação Mundial da Infância 2012: Crianças em um Mundo Urbano. www.unicef.org/brasil/pt/PT.BR_SOWC_2012.pdf. 